\title{
CINÉTICA DE SECAGEM OSMO-CONVECTIVA DE PIMENTÃO VERDE: INFLUÊNCIA DAS VARIÁVEIS DE PROCESSO
}

\author{
T. P. ALVES ${ }^{1}$, G. R. STRÖHER ${ }^{1}$ e J. F. NICOLETI ${ }^{1}$ \\ ${ }^{1}$ Universidade Tecnológica Federal do Paraná, Campus Apucarana, Departamento de \\ Processos Químicos \\ E-mail para contato: nicoleti@utfpr.edu.br
}

\begin{abstract}
RESUMO - O objetivo desta proposta foi avaliar o efeito das variáveis de processo, entre elas da desidratação osmótica - tempo de contato, concentração e temperatura da solução - e da temperatura do ar de secagem sobre a cinética convectiva de fatias de pimentão verde. O preparo da solução osmótica foi fixado nas proporções de alimento/solução e sal/sacarose em 1:10 e 1:6, p/p, respectivamente. O tempo de contato da amostra com a solução foi avaliada em intervalo de 1 a 3 horas, a temperatura da solução entre 30 e $50{ }^{\circ} \mathrm{C}$ e a concentração de sólidos da solução osmótica entre 40 e 70\%. Após a desidratação osmótica, as amostras foram dispostas sobre bandejas e levadas à estufa com circulação forçada nas temperaturas fixas de 40,55 e $70{ }^{\circ} \mathrm{C}$. Pelo planejamento fatorial, foi possível avaliar os efeitos das variáveis independentes sobre os parâmetros cinéticos empregando os modelos de Page e difusional baseado na segunda Lei de Fick. Resultados são apresentados por superfície de resposta e análise de variância.
\end{abstract}

\section{INTRODUÇÃO}

O pimentão é uma fruta de clima tropical rica em constituintes benéficos na dieta humana. Apresenta-se como uma ótima fonte de diversos nutrientes imprescindíveis e essenciais na manutenção diária de nosso organismo, entre eles minerais, fibras e vitaminas, além de proporcionar cor, aroma e sabor em diversas formulações de alimentos. Entretanto, o pimentão in natura, devido ao seu alto conteúdo de umidade, apresenta uma vida útil muito curta por apresentar uma alta atividade de água, favorecendo assim sua rápida deterioração por micro-organismos e reações deterioradoras como químicas e enzimáticas. Contudo, frutos como o pimentão podem ser secos com o propósito de estender sua vida de prateleira, reduzindo assim desperdícios da produção excedente e com vantagens adicionais. Entre elas pode-se citar a elaboração de um produto com maior valor agregado e redução de peso e volume, implicando na necessidade de espaços para o transporte e armazenamento menores, além de reduzir também custos com embalagem (Camargo, 2007; Raupp, 2009).

A secagem de alimentos é uma operação unitária que visa, além de diversificar e modificar os produtos já disponíveis, estender a vida de prateleira, disponibilizando, desta forma, um alimento com suas características preservadas por um longo período de tempo, ainda que armazenado sob temperatura ambiente, sendo este outro grande atrativo em obter alimentos secos por implicar na redução de custos energéticos, já que não há necessidade de 
serem conservados pelo frio. Com essa técnica já são elaborados produtos de alta qualidade para exportação como tâmaras e uva passa (Gomes et al., 2007).

A pesar da contribuição positiva da secagem que visa diminuir a atividade de água do alimento e, desta forma, disponibilizar um produto seguro ao consumidor, também contribui de forma negativa para a qualidade do produto nos aspectos sensorial e nutricional como a caramelização dos açúcares, reações enzimáticas e degradação da pigmentação, além de causar prejuízos às propriedades de reidratação e perdas das vitaminas. Esses efeitos negativos estão relacionados diretamente com o processo de secagem o qual depende da temperatura, tempo e conteúdo de umidade do alimento. O controle desses parâmetros pode ser conseguido com a obtenção da cinética de secagem, sendo que os dados da cinética são úteis na seleção das condições ideais para cada tipo de alimento, contribuindo para a melhoria da qualidade do produto final.

Diversas investigações sugerem a desidratação osmótica, que consiste na imersão de um alimento sólido em uma solução com alta concentração de substâncias solúveis como glicose, sacarose e cloreto de sódio, como um pré-tratamento ao processo de secagem convencional a ar quente, que minimiza as injurias térmica, reduz o teor de água do alimento resultando na redução nos gastos, tempo e energias além de apresentar melhoria na textura, estabilidade da coloração e uma melhor capacidade de reidratação.

A presente proposta visa avaliar as condições de processamento osmo-convectiva de fatias de pimentão verde, entre elas as condições da solução osmótica (temperatura, concentração e tempo de contato) e da temperatura do ar de secagem, empregando metodologia de superfície de resposta e análise de variância (ANOVA).

\section{MATERIAL E MÉTODOS}

\subsection{Matérias Primas e Preparação}

Foi utilizado pimentão verde (Capsicum annuum L.) adquiridos no mercado local de Apucarana-PR poucas horas antes do processamento. A seleção do pimentão foi realizada visualmente, dando preferência aos frutos sadios, sem injúrias em sua superfície, de coloração uniforme e de textura firme. A caracterização do pimentão foi feita pela quantificação do conteúdo de umidade inicial em estufa a $105^{\circ} \mathrm{C}$ por 4 h (AOAC, 1997).

Cada fruto foi cortado em três ou quatro partes no sentido longitudinal, descartando o pedúnculo e as sementes contidas em seu interior. Em seguida, as fatias foram higienizadas com detergente e água corrente, para posteriormente, serem submersas em solução de hipoclorito para desinfecção. Após a higienização, as fatias foram secas em papel absorvente para seguir à desidratação osmótica.

O preparo da solução osmótica foi fixado nas proporções de alimento/solução e $\mathrm{sal} /$ sacarose em 1:10 e 1:6, respectivamente, empregando água destilada. As fatias de pimentão verde e a solução osmótica, de acordo com o planejamento experimental (Tabela 1) e as proporções estabelecidas, foram introduzidas em um elernmeyer de $250 \mathrm{ml}$, em seguida, esta vidraria foi fixada em uma plataforma com garras no interior da incubadora shaker (MARCONI, MA 420-E), com agitação orbital fixada em $250 \mathrm{rpm}$. 
Após a desidratação osmótica, estas amostras foram passadas em água corrente e enxugadas em papel absorvente, em seguida, foram dispostas sobre bandejas perfuradas de aço inoxidável e levadas à secagem em estufa de circulação forçada (SOLAB, SL-102) juntamente com as amostras padrão (pimentão fresco). Em tempos consecutivos, as bandejas foram pesadas para o posterior cálculo do teor de umidade e construção das curvas de cinética de secagem.

\subsection{Planejamento experimental}

Foi proposto um planejamento fatorial $2^{4}$, com três pontos centrais, totalizando 19 condições experimentais. Considerou-se três níveis codificados $(-1,0,+1)$ e quatro fatores como variáveis independentes, que foram a temperatura da solução osmótica $\left(\mathrm{T}_{\mathrm{DO}}\right)$ com os respectivos valores reais de 30,40 e $50{ }^{\circ} \mathrm{C}$; concentração da solução osmótica $\left(\mathrm{C}_{\mathrm{DO}}\right)$ com os valores de 40,55 e $70 \%(\mathrm{~m} / \mathrm{m})$; tempo de contato $\left(t_{D O}\right)$ com a solução fixados em 1,2 e $3 \mathrm{~h}$ e; temperatura do ar de secagem $\left(\mathrm{T}_{\text {ar }}\right)$ fixadas em 40,55 e $70{ }^{\circ} \mathrm{C}$.

\subsection{Modelagem matemática}

Empregou-se dois modelos - solução analítica da segunda Lei de Fick (Eq. 1) e o modelo de Page (Eq. 2) - aos dados experimentais de cinética de secagem de pimentão. Em consideração a solução analítica, assumiu que as fatias de pimentão aproximavam-se a um sólido de placa plana com somente uma face exposta ao ar de secagem, de difusividade constante, variação do volume desprezível e que o efeito do gradiente de temperatura no interior da amostra também fosse desprezível (Crank, 1975): Vale ressaltar que optou-se pela consideração de secagem por apenas uma das faces devido à existência de uma camada superficial serosa encontrada na pele do pimentão, que possivelmente impede a transferência de massa por este lado. Ajustes preliminares do modelo considerando secagem por apenas um lado confirmaram melhores resultados comparado com a solução analítica obtida para a secagem por ambas as faces.

$$
M=\frac{8}{\pi^{2}} \sum_{n=1}^{\infty} \frac{1}{(2 n-1)^{2}} \exp \left[-(2 n-1)^{2} \frac{\pi^{2} D_{e f f} t}{4 L^{2}}\right]
$$

onde $\mathrm{M}=\left(\mathrm{X}-\mathrm{X}_{\mathrm{eq}}\right) /\left(\mathrm{X}_{0}-\mathrm{X}_{\mathrm{eq}}\right)$ é a umidade adimensional, $\mathrm{X}$ a umidade do sólido em base seca, $\mathrm{X}_{\mathrm{eq}}$ a umidade de equilíbrio, $\mathrm{X}_{0}$ a umidade inicial, $\mathrm{D}_{\mathrm{eff}}$ a difusividade efetiva, $\mathrm{t}$ o tempo e L a espessura da amostra.

$$
M=\exp \left(-k t^{n}\right)
$$

Sendo $\mathrm{M}$ a umidade adimensional, $\mathrm{k}$ e $\mathrm{n}$ constantes da equação e t o tempo.

Para a validação da qualidade dos ajustes dos modelos foram considerados o coeficiente de determinação $\left(\mathrm{R}^{2}\right)$, erro relativo médio (MRE) (Eq. 3) e root mean square (RMS) (Eq. 4). 
$M R E=\frac{100}{N} \times \sum_{i=1}^{N} \frac{\left|X_{e}-X_{t}\right|}{X_{e}}$

$M R S=100 \times \sqrt{\frac{\sum_{i=1}^{N}\left[\left(\frac{X_{e}-X_{t}}{X_{e}}\right)^{2}\right]}{N}}$

onde $\mathrm{X}_{\mathrm{t}}$ representam os valores teóricos, $\mathrm{X}_{\mathrm{e}}$ os valores experimentais das umidades de equilíbrio e $\mathrm{N}$ o número total pontos.

Para verificar o efeito das variáveis independentes do processo $\left(T_{A R}, T_{D O}, C_{D O}\right.$ e $\left.t_{D O}\right)$ sobre os parâmetros cinéticos avaliou-se os resultados por análise de variância (ANOVA) considerando os efeitos significativos $(p<0,05)$ e pela construção de superfícies de resposta (MSR) empregando o software Statistica 7.0.

\section{RESULTADOS E DISCUSSÃO}

Para cada ensaio realizado, a Tabela 1 traz os valores estimados de difusividade efetiva, obtida pela Eq. (1) considerando os quatro primeiros termos da série, bem como os respectivos valores de $\mathrm{R}^{2}$, MRE e RMS. Observa-se que apesar dos coeficientes de correlação $\left(R^{2}\right)$ serem superiores a 0,93 , a avaliação dos demais fatores estatísticos apresentam desvios consideráveis. Possivelmente a discrepância se dá pelas considerações de se assumir a difusividade como um parâmetro constante e negligenciar o fenômeno de encolhimento durante o processo.

Tabela 1 - Difusividade efetiva da água em função das variáveis de processo.

\begin{tabular}{ccccccccc}
\hline $\begin{array}{c}\text { Espessura } \\
(\mathrm{mm})\end{array}$ & $\begin{array}{c}\mathrm{T}_{\mathrm{AR}} \\
\left({ }^{\circ} \mathrm{C}\right)\end{array}$ & $\begin{array}{c}\mathrm{T}_{\mathrm{DO}} \\
\left({ }^{\circ} \mathrm{C}\right)\end{array}$ & $\begin{array}{c}\mathrm{t}_{\mathrm{DO}} \\
(\mathrm{h})\end{array}$ & $\mathrm{C}_{\mathrm{DO}}(\mathrm{m} / \mathrm{m})$ & $\begin{array}{c}\mathrm{D}_{\text {eff }} \\
\left(\mathrm{m}^{2} \cdot \mathrm{s}^{-1}\right) \cdot 10^{10}\end{array}$ & $\mathrm{R}^{2}$ & $\mathrm{MRE} \%$ & $\mathrm{RMS} \%$ \\
\hline $5,19 \pm 0,4$ & 40 & 30 & 1 & 40 & 1,1689 & 0,965 & 28,26 & 54,20 \\
$4,87 \pm 0,1$ & 40 & 30 & 3 & 40 & 1,2336 & 0,972 & 27,02 & 52,10 \\
$4,89 \pm 0,1$ & 40 & 30 & 1 & 70 & 0,9483 & 0,966 & 19,80 & 33,53 \\
$5,49 \pm 0,9$ & 40 & 30 & 3 & 70 & 0,9758 & 0,966 & 25,29 & 42,90 \\
$4,66 \pm 0,5$ & 40 & 50 & 1 & 40 & 1,2990 & 0,968 & 20,36 & 38,45 \\
$4,78 \pm 1,5$ & 40 & 50 & 3 & 40 & 1,7300 & 0,976 & 17,71 & 26,25 \\
$4,39 \pm 0,4$ & 40 & 50 & 1 & 70 & 1,2760 & 0,977 & 16,14 & 28,28 \\
$5,23 \pm 0,3$ & 40 & 50 & 3 & 70 & 1,4890 & 0,966 & 39,28 & 86,19 \\
$4,82 \pm 1,1$ & 55 & 40 & 2 & 55 & 2,7090 & 0,977 & 35,70 & 73,89 \\
$4,54 \pm 1,8$ & 55 & 40 & 2 & 55 & 2,5380 & 0,980 & 10,64 & 33,57 \\
$5,14 \pm 0,6$ & 55 & 40 & 2 & 55 & 2,9620 & 0,991 & 32,36 & 49,63 \\
$4,70 \pm 0,1$ & 70 & 30 & 1 & 40 & 6,7433 & 0,963 & 246,78 & 469,35 \\
$4,59 \pm 0,1$ & 70 & 30 & 3 & 40 & 7,1267 & 0,957 & 397,92 & 756,08 \\
$4,56 \pm 0,8$ & 70 & 30 & 1 & 70 & 7,1842 & 0,987 & 251,64 & 457,25 \\
$4,66 \pm 0,3$ & 70 & 30 & 3 & 70 & 7,8425 & 0,966 & 333,80 & 822,93
\end{tabular}




\begin{tabular}{ccccccccc}
$4,81 \pm 0,3$ & 70 & 50 & 1 & 40 & 6,1900 & 0,965 & 553,36 & 1042,00 \\
$5,10 \pm 0,3$ & 70 & 50 & 3 & 40 & 5,6650 & 0,959 & 316,00 & 930,95 \\
$4,78 \pm 0,2$ & 70 & 50 & 1 & 70 & 5,2890 & 0,937 & 826,85 & 1689,30 \\
$4,98 \pm 0,1$ & 70 & 50 & 3 & 70 & 7,5635 & 0,967 & 155,33 & 287,52 \\
\hline
\end{tabular}

Na Tabela 2 encontram-se os parâmetros K e n da equação de Page (Eq. 2) para cada tratamento delineado no planejamento experimental, e os valores dos parâmetros estatísticos $\mathrm{R}^{2}$, MRE e RMS utilizados para avaliar a adequação do modelo matemático. Os ajustes do modelo de Page descreveram melhor a cinética de secagem das fatias de pimentão verde observado pelos $\mathrm{R}^{2}$ superiores a 0,99 e pelos menores desvios calculados pelos MRE e RMS quando comparado pela avaliação estatística empregando o modelo difusional da $2^{\mathrm{a}}$ Lei de Fick. Os parâmetros $\mathrm{K}$ e $\mathrm{n}$ têm sido correlacionados com diferentes variáveis do processo (temperatura, umidade inicial, entre outros). Este modelo empírico deriva diretamente da relação entre o conteúdo de umidade médio e o tempo de secagem (Argandoña, 2005).

Tabela 2 - Parâmetros da equação de Page (k e n) em função das variáveis de processo.

\begin{tabular}{|c|c|c|c|c|c|c|c|c|}
\hline $\begin{array}{l}\mathrm{T}_{\mathrm{AR}} \\
\left({ }^{\circ} \mathrm{C}\right)\end{array}$ & $\begin{array}{l}\mathrm{T}_{\mathrm{DO}} \\
\left({ }^{\circ} \mathrm{C}\right)\end{array}$ & $\begin{array}{l}t_{\mathrm{DO}} \\
\text { (h) }\end{array}$ & $\begin{array}{c}\mathrm{C}_{\mathrm{DO}} \\
(\mathrm{m} / \mathrm{m})\end{array}$ & Parâmetros & s - Page & $\mathrm{R}^{2}$ & MRE\% & $\mathrm{RMS} \%$ \\
\hline & & & & K.10 & $\mathrm{n}$ & & & \\
\hline 40 & 30 & 1 & 40 & 0,8 & 0,8644 & 0,998 & 1,16 & 5,95 \\
\hline 40 & 30 & 3 & 40 & 1,2 & 0,8333 & 0,997 & 1,9 & 8,43 \\
\hline 40 & 30 & 1 & 70 & 1,1 & 0,8284 & 0,998 & 4,19 & 5,70 \\
\hline 40 & 30 & 3 & 70 & 1,1 & 0,8251 & 0,998 & 1,73 & 4,40 \\
\hline 40 & 50 & 1 & 40 & 0,9 & 0,8660 & 0,999 & 2,32 & 3,51 \\
\hline 40 & 50 & 3 & 40 & 1,5 & 0,8249 & 0,999 & 1,86 & 2,66 \\
\hline 40 & 50 & 1 & 70 & 1,4 & 0,8207 & 0,998 & 2,3 & 4,41 \\
\hline 40 & 50 & 3 & 70 & 0,7 & 0,8872 & 0,996 & 6,48 & 17,55 \\
\hline 55 & 40 & 2 & 55 & 1,2 & 0,8943 & 0,997 & 1,73 & 2,38 \\
\hline 55 & 40 & 2 & 55 & 2,7 & 0,7852 & 0,998 & 1,1 & 1,43 \\
\hline 55 & 40 & 2 & 55 & 6,5 & 0,7192 & 0,996 & 2,17 & 2,86 \\
\hline 70 & 30 & 1 & 40 & 0,3 & 1,1005 & 0,997 & 37,07 & 74,21 \\
\hline 70 & 30 & 3 & 40 & 0,2 & 1,1685 & 0,994 & 36,31 & 72,31 \\
\hline 70 & 30 & 1 & 70 & 1,9 & 0,9279 & 0,998 & 19,53 & 40,74 \\
\hline 70 & 30 & 3 & 70 & 0,4 & 1,0908 & 0,998 & 32,15 & 65,03 \\
\hline 70 & 50 & 1 & 40 & 0,4 & 1,0898 & 0,994 & 55,14 & 106,90 \\
\hline 70 & 50 & 3 & 40 & 0,2 & 1,1254 & 0,995 & 66,67 & 116,6 \\
\hline 70 & 50 & 1 & 70 & 0,05 & 1,3024 & 0,995 & 43,69 & 100,40 \\
\hline 70 & 50 & 3 & 70 & 0,4 & 1,0918 & 0,994 & 31,2 & 47,89 \\
\hline
\end{tabular}

$\mathrm{O}$ efeito das variáveis independentes $\left(\mathrm{T}_{\mathrm{AR}}, \mathrm{T}_{\mathrm{DO}}, \mathrm{C}_{\mathrm{DO}}, \mathrm{t}_{\mathrm{DO}}\right)$ sobre as variáveis respostas difusividade efetiva e constantes da equação de Page - foi avaliado por meio de superfície de 
resposta, aplicando análise de variância (ANOVA) com significância de $95 \%(p<0,05)$. De acordo com a Tabela 3, somente o efeito linear da temperatura do ar ( $\left.\mathrm{T}_{\mathrm{AR}}\right)$ foi significativo $(\mathrm{p}<0,05)$ sobre os valores da difusividade efetiva e do parâmetro $\mathrm{n}$ de Page. Em relação ao parâmetro k, este não apresentou uma tendência definida, sendo que nenhuma das variáveis independentes avaliadas teve feito significativo sobre seus valores $(p<0,05)$.

Tabela 3 - Nível de significância dos fatores independentes sobre os parâmetros $D_{\text {eff }}$ e $n$

\begin{tabular}{ccc}
\hline Efeitos & \multicolumn{1}{c}{$\mathrm{D}_{\text {eff }}$} & $\mathrm{n}$ \\
\cline { 2 - 3 } & \multicolumn{2}{c}{$\mathrm{p}<0,05$} \\
\hline $\mathbf{T}_{\mathrm{AR}}$ & $\mathbf{0 , 0 0 0 0 0 3}$ & $\mathbf{0 , 0 0 3 5}$ \\
$\mathrm{T}_{\mathrm{DO}}$ & 0,859268 & 0,5028 \\
$\mathrm{t}_{\mathrm{DO}}$ & 0,978165 & 0,9312 \\
$\mathrm{C}_{\mathrm{DO}}$ & 0,965919 & 0,8562 \\
$\mathrm{~T}_{\mathrm{AR}} \times \mathrm{T}_{\mathrm{DO}}$ & 0,566445 & 0,6166 \\
$\mathrm{~T}_{\mathrm{AR}} \times \mathrm{t}_{\mathrm{DO}}$ & 0,719548 & 0,9049 \\
$\mathrm{~T}_{\mathrm{AR}} \times \mathrm{C}_{\mathrm{DO}}$ & 0,770469 & 0,9353 \\
$\mathrm{~T}_{\mathrm{DO}} \times \mathrm{t}_{\mathrm{DO}}$ & 0,922862 & 0,5293 \\
$\mathrm{~T}_{\mathrm{DO}} \times \mathrm{C}_{\mathrm{DO}}$ & 0,847975 & 0,3787 \\
$\mathrm{t}_{\mathrm{DO}} \times \mathrm{C}_{\mathrm{DO}}$ & 0,685699 & 0,9766 \\
\hline
\end{tabular}

Os modelos matemáticos que descrevem os comportamentos da difusividade efetiva e do parâmetro n, de Page, em função da temperatura do ar de secagem (Tar), calculado pelo software Statistica 7.0, são representados pelas Equações (5) e (6), desconsiderando o efeito das demais variáveis independentes $\left(\mathrm{T}_{\mathrm{DO}}, \mathrm{C}_{\mathrm{DO}}, \mathrm{t}_{\mathrm{DO}}\right)$ por não apresentarem resultados significativos $(\mathrm{p}<0,05)$.

$D_{\text {eff }}=-6,0998 \times 10^{-10}+0,2141 \times 10^{-10} \times T_{A R}$

$\mathrm{n}=-0,895+0,000451 \times \mathrm{T}_{\mathrm{AR}}$

De forma ilustrativa, as Figuras 1 e 2 apresentam as superfícies de resposta da difusividade efetiva e do parâmetro $\mathrm{n}$ de Page, em função da temperatura do ar e da concentração da solução osmótica, respectivamente. Como esperado pelos resultados da ANOVA, observa-se somente o efeito da temperatura do ar sobre a difusividade efetiva, evidenciando um aumento em seus valores conforme a temperatura é aumentada. 

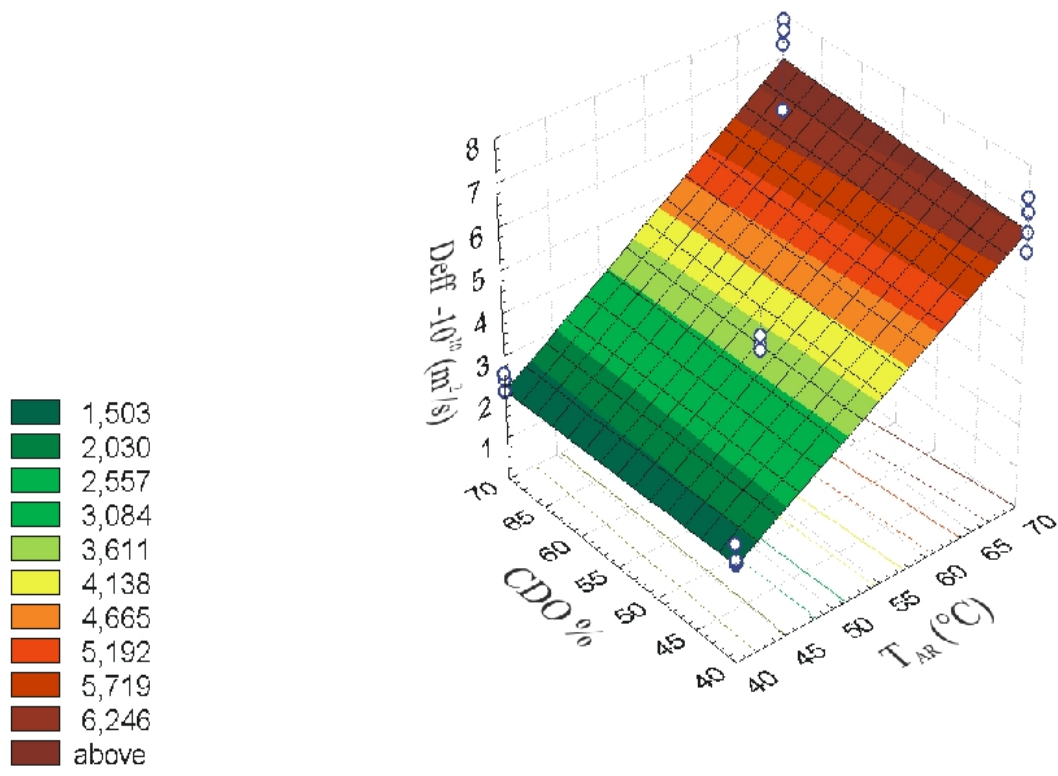

Figura 1 - Superfícies de resposta para a difusividade efetiva em função da temperatura do ar e concentração da solução osmótica.
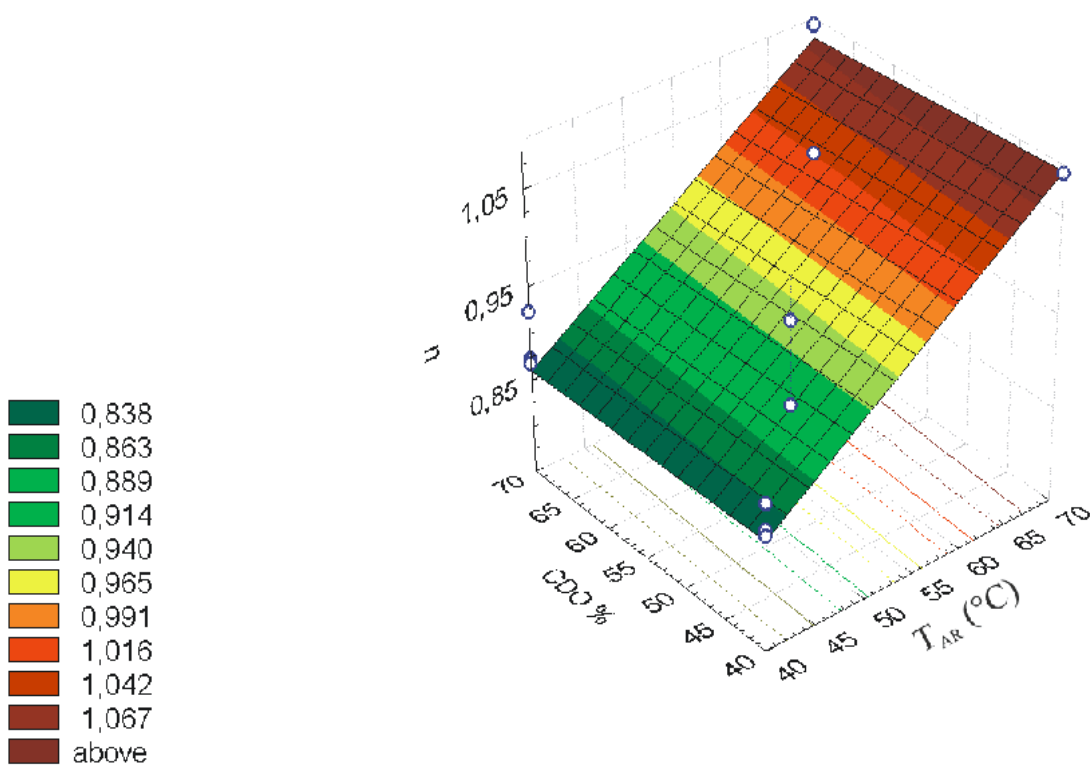

Figura 2 - Superfícies de resposta para o parâmetro n de Page em função da temperatura do ar e concentração da solução osmótica. 


\section{CONCLUSÕES}

A temperatura do ar de secagem foi à única variável significativa do processo sobre os parâmetros $\mathrm{D}_{\text {eff }}$ do modelo de Fick e $\mathrm{n}$ da equação de Page, de acordo com a avaliação ANOVA. Os modelos difusional de Fick e de Page apresentaram bons ajustes aos dados experimentais de secagem em todos os tratamentos realizados, sendo os melhores ajustes obtidos pelo modelo de Page, conforme a avaliação de qualidade dos parâmetros. Os valores da divusividade efetiva e da potência "n" da equação de Page tiveram seus valores acrescidos conforme a temperatura de secagem foi aumentada.

\section{AGRADECIMENTOS}

Os autores agradecem a Fundação Araucária pelo Auxílio Financeiro (Conv. 469/2010, Projeto 16.742) e pela Bolsa de Iniciação Científica (PIBIC 20010/2011 e 2012/2013).

\section{REFERÊNCIAS}

AOAC - Association of Official Analytical Chemists. Official Methods of Analysis,16 ed. Association of Official Analytical Chemists, v. 1, p. 40, 1997.

ARGANDOÑA, E. J. S. Goiabas desidratadas osmoticamente e secas: Avaliação de um sistema osmótico semicontínuo, da secagem e da qualidade. Tese (Doutorado em Engenharia de Alimentos), Faculdade de Engenharia de Alimentos, Universidade Estadual de Campinas, São Paulo, 2005.

CAMARGO, G. A. Avaliação da qualidade de tomate seco em conserva. Revista Brasileira de Engenharia Agrícola e Ambiental, Campina Grande, v. 11, n. 5, p. 521-526, 2007.

CRANK, J. The mathematics of diffusion. Pergamon Press, Oxford, 1975.

GOMES, A. T.; CEREDA, M. P.; VILPOUX, O. Desidratação osmótica: uma tecnologia de baixo custo para o desenvolvimento da agricultura familiar. Revista Brasileira de Gestão e Desenvolvimento Regional, v. 3, n. 3, p.212-226, 2007.

RAUPP, D. S. Processamento de tomate seco de diferentes cultivares. Acta Amazônica, Manaus, v. 39, n. 2, p. 415-422, 2009. 